\title{
A Compact Rectenna Using Split Ring Resonator for Energy Harvesting
}

\author{
Khaled Aljaloud \\ Electronic and Electrical Engineering Department \\ Roberts Building, University College London \\ Torrington Place, London \\ WC1E 7JE \\ Khaled.aljaloud.15@ucl.ac.uk
}

\author{
Kin-Fai Tong \\ Electronic and Electrical Engineering Department \\ Roberts Building, University College London \\ Torrington Place, London \\ WC1E 7JE \\ k.tong@ucl.ac.uk
}

\begin{abstract}
In this paper, a rectifying antenna circuit based on the concept of electrically small resonator is reported. To verify the concept, it is designed, simulated, and also tested to harvest electromagnetic energy at $3.1 \mathrm{GHz}$. It is observed through measurements that the proposed circuit is able to transfer $18 \%$ of the RF incident power into DC power. The DC output power and DC voltage are investigated with respect to frequency and also rotation of the proposed design.
\end{abstract}

\section{INTRODUCTION}

Harvesting and recycling the energy from the electromagnetic waves, commonly refers as rectenna or Wireless Power Transfer (WPT). In order to achieve high efficient and sustainable energy production systems, many techniques have been proposed in the literature [1]-[3]. Patch antennas can be used as $t$ he absorber or collector of the energy in the rectifier circuits. This results in increasing the overall size of the circuit. To overcome this problem, a collector based on metamaterial cell to collect electromagnetic energy, which has small size and high efficiency compared with patch antennas, has been proposed in [4].

The number of energy collectors in the energy harvesting system is critical to improve the system efficiency of transferring the incident RF energy into DC Power. Therefore, having a small size collector is in demand for increase the number of energy collectors in such a system. As a result, a Split Ring Resonator (SRR) is used as the collector of energy in our work. However, only RF-to-AC power was reported in [4]. In this work, SRR is integrated with a rectification circuit in order to transfer the collected RF power into DC power.

\section{DESIGN AND RESULTS}

The SRR in this work is designed to resonate at $3.1 \mathrm{GHz}$. It is built on a FR-4 substrate $\left(\epsilon_{r}=4.1, \tan \delta=0.018\right.$ and thickness $=1.6 \mathrm{~mm}$ ). A full wave simulator $(\mathrm{CST})$ is used to simulate the performance of the proposed system. The parameters from the data sheet of the diode were used in the simulation. Fig. 1(a) shows the proposed rectenna system based on the SRR as a collector element to receive incident $\mathrm{RF}$ power. The length of each side of SRR is $\mathrm{L}=7.6 \mathrm{~mm}$ and the width of the line is $\mathrm{W}=0.55 \mathrm{~mm}\left(4 \mathrm{~L} \approx 0.31 \lambda_{o}\right)$. The overall size of the rectenna system is $20 \times 28 \mathrm{~mm}^{2}$. The fabricated prototype of the proposed rectenna system is shown in Fig. 1(b). The collector and the rectifying circuit are printed and mounted on the same side of the substrate, which reduces the complexity of the system and also make the integration of the rectenna system to other devices simpler.

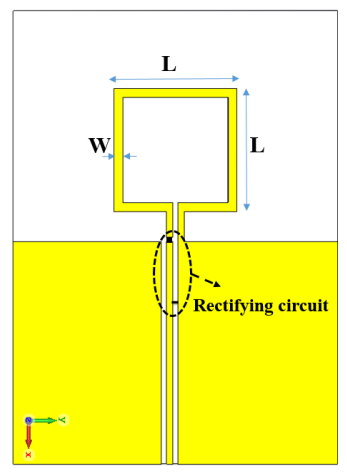

(a)

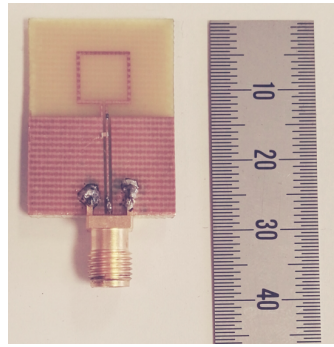

(b)
Fig. 1. The proposed rectenna system.

An experimental setup, as shown in Fig. 2, is used to verify the proposed rectenna system. A Satimo Quad Ridge Referene horn antenna $(800 \mathrm{MHz}-12 \mathrm{GHZ})[5]$, which has a gain of $11 \mathrm{dBi}$ at the operating frequency, is used as the source of electromagnetic power in the experiment. The signal generator is set to transmit from $2.7 \mathrm{GHz}$ to $3.7 \mathrm{GHz}$, and it feeds the antenna with $7.5 \mathrm{dBm}$ as an input power. The distance between the horn antenna and rectenna system is about 10 $\mathrm{cm}$. The system is terminated by a load of $50 \Omega$ The Tektronix oscilloscope is used to measure the DC output voltage across the load. The measured DC voltage of the proposed circuit is about $16.5 \mathrm{mV}$.

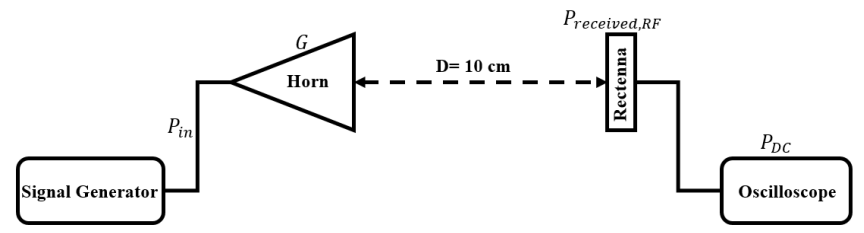

Fig. 2. Experimental setup to measure the DC output voltage. 
The measured result is compared with the result obtained from CST as shown in Fig. 3(a,b). The RF-to-DC conversion efficiency depends on the input power, the type of diode, and the load. The area of the collector is multiplied by incident power intensity (S) using Eq. (1) [6]. Eq. (2) is used to calculate the total efficiency of the rectenna system:

$$
\begin{gathered}
S=r_{0} \frac{P_{i n} G}{4 \pi D^{2}} \\
\eta=\frac{P_{D C}}{P_{\text {received }, R F}}
\end{gathered}
$$

Where $P_{i n}$ is the input power and $r_{0}$ is the directivity. G and $\mathrm{D}$ are the gain and the distance between the horn antenna and rectenna, respectively. In Eq. (2), $P_{D C}$ and $P_{\text {received,RF }}$ is the maximum DC output power obtaind by rectenna and the received RF power available at the footprint of the collector element, respectively.

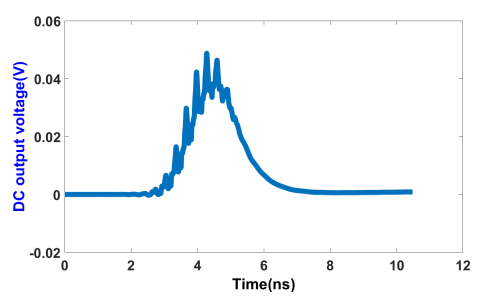

(a)

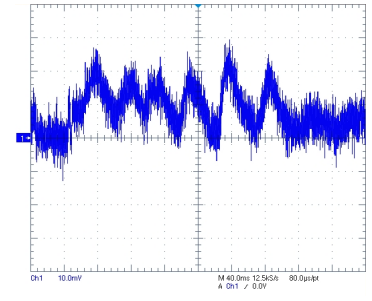

(b)
Fig. 3. DC output voltage signal across the load obtaind from: (a) CST. (b)Oscillicope.

Harvesting of continues waves (CW) is tested at the operating frequency and other frequencies, the DC output power is measured for each frequency, and the RF-to-DC efficiency is computed by means of Eq. (2). The maximum DC power is $5.44 \mu \mathrm{W}$ at $3.1 \mathrm{GHz}$. However, the proposed system is able to harvest the electromagnetic radiation at other frequencies. The results are listed in Table I.

TABLE I: DC output power and RF-to-DC efficiency

\begin{tabular}{|c||c|c|}
\hline Frequency $(\mathrm{GHz})$ & DC output Power $(\mu \mathrm{W})$ & Efficiency $(\%)$ \\
\hline 2.7 & 0.32 & 1.07 \\
\hline 2.9 & 1.04 & 3.45 \\
\hline 3.1 & 5.44 & 18 \\
\hline 3.3 & 0.62 & 2.1 \\
\hline 3.5 & 0.62 & 2.1 \\
\hline 3.7 & 0.29 & 1 \\
\hline
\end{tabular}

Since the proposed system is not required a ground plane on the back, the DC output power and the efficiency are investigated with respect to the rotation of the system as illustrated in Fig. 4. The proposed rectenna is able to harvest the incident waves when the rectenna rotated around y axis 180 degree with DC output power of $4.68 \mu \mathrm{W}$. The relationship between the input power level and rectified power is displayed in Fig. 5.

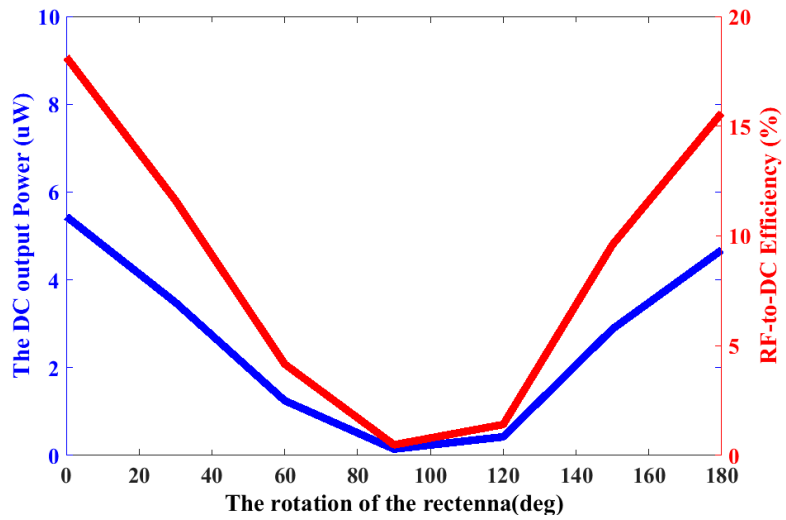

Fig. 4. The DC voltage and the RF-to-DC conversion efficiency with respect to the rotation of the rectenna.

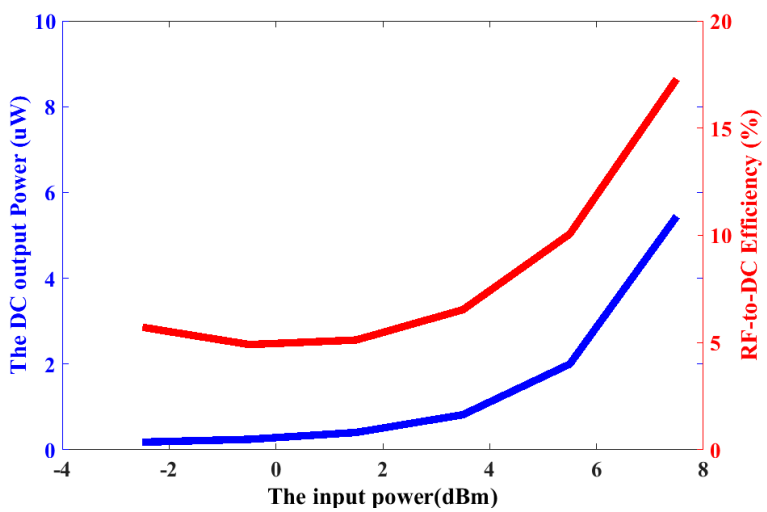

Fig. 5. The DC voltage and the output power with respect to input powr level.

\section{CONCLUSION}

A compact unit cell of energy harvesting system used split ring resonator (SRR) is designed, implemented, and then verified. The footprint of the system is reduced by means of printing the SRR and rectifying circuit on the same side of the substrate. The rectenna is evaluated, and results display that about $5.44 \mu \mathrm{W}$ and RF-to-DC conversion efficiency of $18 \%$ are achieved.

\section{REFERENCES}

[1] J. A. Hagerty, F. B. Helmbrecht, W. H. McCalpin, R. Zane and Z. B. Popovic, "Recycling ambient microwave energy with broad-band rectenna arrays," in IEEE Transactions on Microwave Theory and Techniques, vol. 52, no. 3, pp. 1014-1024, March 2004.

[2] T. S. Almoneef and O. M. Ramahi, "3-D stacked antenna panels: The promise of high efficient energy harveters," 2016 IEEE International Symposium on Antennas and Propagation (APSURSI), Fajardo, 2016, pp. 601-602.

[3] Young-Ho Suh and Kai Chang, "A high-efficiency dual-frequency rectenna for 2.45- and 5.8-GHz wireless power transmission," in IEEE Transactions on Microwave Theory and Techniques, vol. 50, no. 7, pp. 1784-1789, Jul 2002.

[4] T. Almoneef and O. M. Ramahi, Split-Ring Resonator Arrays for Electromagnetic Energy Harvesting, Prog. Electromagn.Res., vol. 62, 167180, 2015 .

[5] www.satimo.com, Ref : TR.017.2.11.SATI. $B_{Q} H 800$

[6] C. A. Balanis, Antenna Thorey: Analysis and Design, 3rd ed., Hoboken, NJ, USA: Wiley, 2016. 